\title{
ESTRUTURA E COMPOSIÇÃO FLORÍSTICA DO ESTRATO ARBÓREO DE UM REMANESCENTE DE MATA ATLÂNTICA SUBMONTANA NO MUNICÍPIO DE RIO BONITO, RJ, BRASIL (MATA RIO VERMELHO) ${ }^{1}$
}

\author{
Fabrício Alvim Carvalho ${ }^{2}$, Marcelo Trindade Nascimento ${ }^{2}$ e João Marcelo Alvarenga Braga ${ }^{2,3}$
}

\begin{abstract}
RESUMO - Neste trabalho, descreveram-se a composição florística e a estrutura do estrato arbóreo de um remanescente de Floresta Ombrófila Densa Submontana (Mata Rio Vermelho) na região Centro-Norte fluminense, comparando-a com outras florestas da região. Foram alocadas oito parcelas de $5 \mathrm{~m}$ x $100 \mathrm{~m}$, e todos os indivíduos vivos e mortos com DAP $\geq 5 \mathrm{~cm}$ foram amostrados. Ao todo, foram registradas 106 espécies pertencentes a 77 gêneros e 32 famílias. As famílias com maior riqueza de espécies foram Leguminosae (13 espécies) e Lauraceae (8), e as mais abundantes foram Monimiaceae (13\% dos indivíduos) e Leguminosae (11\%). As espécies mais importantes quanto ao valor de cobertura (VC) foram Siparuna guianensis, Apuleia leiocarpa, Cupania oblongifolia e Machaerium brasiliensis, todas características de áreas secundárias. O índice de diversidade de espécies $\left(\mathrm{H}^{\prime}=3,91\right.$ nats.ind $\left.^{-1}\right)$ foi próximo ao encontrado em outras florestas secundárias. Os resultados (elevado número de árvores mortas, com lianas, perfilhadas e secundárias iniciais; baixo número de árvores de grande porte e área basal) indicaram que a mata em foco se encontrava perturbada e em fase de regeneração intermediária. Ainda assim, permanecia detentora de considerável riqueza e diversidade florística, com espécies arbóreas ameaçadas de extinção, como Melanoxylon brauna e Dalbergia nigra. Devido à importância ecológica desde remanescente para a manutenção da flora e fauna local e ao avançado processo de fragmentação da região, sugere-se que a Mata Rio Vermelho seja prioritária em programas de conservação e manejo.
\end{abstract}

Palavras-chave: Mata Atlântica, floresta secundária e fitossociologia.

\section{STRUCTURE AND FLORISTIC COMPOSITION OF A TREE COMMUNITY IN A SUBMONTANE ATLANTIC FOREST REMNANT IN RIO BONITO, RJ, BRAZIL (RIO VERMELHO FOREST)}

\begin{abstract}
This work describes the floristic composition and forest structure of a tree community in a Submontane Ombrofilous Dense Atlantic forest (Rio Vermelho Forest) and compares this forest to other remnant forests in the region. Eight $100 \mathrm{~m} \times 5 \mathrm{~m}$ plots were allocated and all trees $\geq 5 \mathrm{~cm}$ DBH were sampled. A total of 106 species was sampled, distributed in 77 genus and 32 families. The richest families in number of species were Leguminosae (13 species) and Lauraceae (8). Monimiaceae (13\% of trees) and Leguminosae (11\%) had the highest densities. The most important species (cover value) were Siparuna guianensis, Apuleia leiocarpa, Cupania oblongifolia and Machaerium brasiliensis. These species are common in secondary Atlantic forests. The species diversity $\left(H^{\prime}=3,91\right.$ nats. ind $\left.^{-1}\right)$ can be considered within the values found for secondary Atlantic forests. Based on the floristic data and the high values found for standing dead trees, trees supporting lianas, number of multiple stems and number of early secondary species and the low number of large trees, this
\end{abstract}

\footnotetext{
${ }^{1}$ Recebido em 02.01.2006 e aceito para publicação em 29.03.2007

${ }^{2}$ Laboratório de Ciências Ambientais (LCA/CBB) da Universidade Estadual do Norte Fluminense (UENF). Av. Alberto Lamego, 2000, Pq. Califórnia. 28015-620 Campos dos Goytacazes-RJ, Brasil. E-mail: <fabricioalvim@yahoo.com.br>.

${ }^{3}$ Instituto de Pesquisas do Jardim Botânico do Rio de Janeiro, Rua Pacheco Leão, 915. 22460-030 Rio de Janeiro-RJ, Brasil.
} 


\begin{abstract}
forest can be classified as a secondary forest in an intermediate regenerating status. However, this forest still has a considerable richness and diversity with some endangered tree species such as Melanoxylon brauna and Dalbergia nigra. Due to its ecological importance for the local flora and fauna and the fragmentation process in the region, this remnant forest should be considered as a priority area for establishment of conservation and management practices.
\end{abstract}

Keywords: Atlantic Forest, secondary forest and phytosociology.

\section{INTRODUÇÃO}

A Mata Atlântica do Estado do Rio de Janeiro, que no passado cobria $100 \%$ do seu território, encontrase hoje restrita a menos de $20 \%$, cujas maiores manchas são observadas nas vertentes das cadeias montanhosas da Serra do Mar, onde a altitude geralmente ultrapassa a cota de $500 \mathrm{~m}$ (FUNDAÇÃO SOS MATAATLÂNTICA, 2002). A região da planície litorânea do Centro-Norte Fluminense, coberta em sua maior parte pela Floresta Ombrófila Densa de Terras Baixas e Submontana (VELOSO et al., 1991), durante séculos foi alvo de intensas perturbações antrópicas, intensificadas nas últimas sete décadas, através da extração madeireira ou da substituição de suas florestas por áreas agrícolas (DEAN, 1996). A paisagem atual da região é constituída por fragmentos florestais geralmente pequenos, isolados, perturbados e circundados por extensas matrizes antrópicas, como pastos e monoculturas (FUNDAÇÃO SOS MATA ATLÂNTICA, 2002).

Estudos na região Centro-Norte fluminense vêm indicando que diversos fragmentos de Floresta Ombrófila Densa Submontana, mesmo aqueles com tamanhos reduzidos e com longo histórico de perturbação, ainda se mantêm detentores de grande representatividade florística(GUEDES-BRUNI, 1998; NEVES, 1999; BORÉM eOLIVEIRA-FILHO, 2002; PESSOA, 2003; RODRIGUES, 2004; CARVALHO, 2005). Entretanto, em termos estruturais muitos desses remanescentes encontramse desprotegidos da contínua ação antrópica predatória, principalmente aqueles localizados em propriedades privadas.

Atualmente, alguns desses remanescentes foram transformados em Unidades de Conservação, representados principalmente pelas Reservas Biológicas Federais de Poço das Antas (cerca de 5.500 ha) e União (cerca de 3.200 ha), além de diversas Reservas Particulares do Patrimônio Natural-RPPN (CARVALHO et al., 2004). Entretanto, a proteção dos remanescentes por si só não garante a sua conservação, tendo em vista a necessidade, em alguns casos, da utilização de práticas de manejo e enriquecimento florestal, objetivando auxiliar o processo de regeneração (VIANA e TABANEZ, 1996).

Ressalta-se que o estudo promovido pelo Ministério de Meio Ambiente (BRASIL, 2000), no que tange à identificação de áreas prioritárias para a conservação da biodiversidade da Mata Atlântica, apontou essa região como de extrema importância ecológica para a flora e fauna. Estudos sobre a estrutura e composição florística de seus remanescentes são necessários, dada a considerável diversidade de espécies da flora local em contraposição com a crescente perda na qualidade de habitat e diminuição da extensão de áreas com florestas naturais.

Nesse contexto, os objetivos deste trabalho foram: 1) avaliar a estrutura e composição florística do componente arbóreo de um remanescente de Floresta Ombrófila Densa Submontana em propriedade privada e com cerca de 800 ha no Município de Rio Bonito, RJ; e 2) compará-la a uma floresta preservada de mesma fitofisionomia (Floresta Ombrófila Densa Submontana) na região. Dessa maneira, buscou-se também disponibilizar informações pertinentes a futuros projetos de manejo, recuperação e preservação dos remanescentes florestais da região Centro-Norte fluminense.

\section{MATERIAL E MÉTODOS}

\section{1. Área de estudo}

A mata Rio Vermelho possui uma área com cerca de 800 ha de Floresta Ombrófila Densa Submontana (VELOSO et al., 1991), situada em propriedade privada no Município de Rio Bonito, região Centro-Norte do Estado do Rio de Janeiro (Figura 1). Essa mata constitui um dos maiores remanescentes de Floresta Ombrófila Densa Submontana da Bacia do Rio São João e apresenta grande importância como habitat do mico-leão-dourado (Leontopithecus rosalia), primata ameaçado de extinção (IBAMA, 1992). Esse remanescente encontra-se em 
propriedade particular e, ainda, suscetível a atividades antrópicas, como corte seletivo de madeira, caça e queimadas. Sua topografia é predominantemente de planície costeira sobre morros e morrotes arredondados (morrotes mamemolares), que não ultrapassam os 150 $\mathrm{m}$ de altitude. Predomina na região o clima tropical úmido com estação chuvosa no verão e sem inverno pronunciado, estando enquadrado pela classificação de Köppen no tipo As tropical chuvoso com estação seca no inverno. A precipitação pluviométrica oscila entre 1.500 e $2.000 \mathrm{~mm}$, sendo os meses de novembro a março os mais chuvosos e de maiores temperaturas (PRIMO e VÖLKER, 2003).

\subsection{Análise da vegetação}

Para a análise da vegetação, utilizou-se o método de parcelas dispostas de formas longitudinais, visando à obtenção de maior heterogeneidade florística e diminuindo, assim, as chances de a amostragem permanecer restrita a uma mancha vegetal (CAUSTON, 1988). Ao longo do remanescente foram estabelecidas, aleatoriamente, oito parcelas de $100 \mathrm{~m}$ x $5 \mathrm{~m}$ no sentido norte-sul, distantes pelo menos $100 \mathrm{~m}$ umas das outras, totalizando uma área amostral de $4.000 \mathrm{~m}^{2}$ (0,4 ha). Em cada parcela, todas as árvores vivas e mortas em pé com $\mathrm{DAP} \geq 5,0 \mathrm{~cm}$ (diâmetro à altura do peito $=1,30 \mathrm{~m}$ do solo) foram amostradas e medidas quanto à altura e ao DAP. Apenas os indivíduos vivos foram plaqueados. Indivíduos com troncos múltiplos ou cespitosos foram amostrados somente quando pelo menos uma das ramificações possuía $\mathrm{DAP} \geq 5,0 \mathrm{~cm}$, e, nesse caso, todas foram medidas, e a área basal total do indivíduo foi calculada pelo somatório das áreas basais de cada ramo. Em cada indivíduo foi verificada a ocorrência e o número de lianas que utilizavam-no como suporte. O período de coleta de dados no campo estendeu-se de novembro de 2002 a junho de 2003.

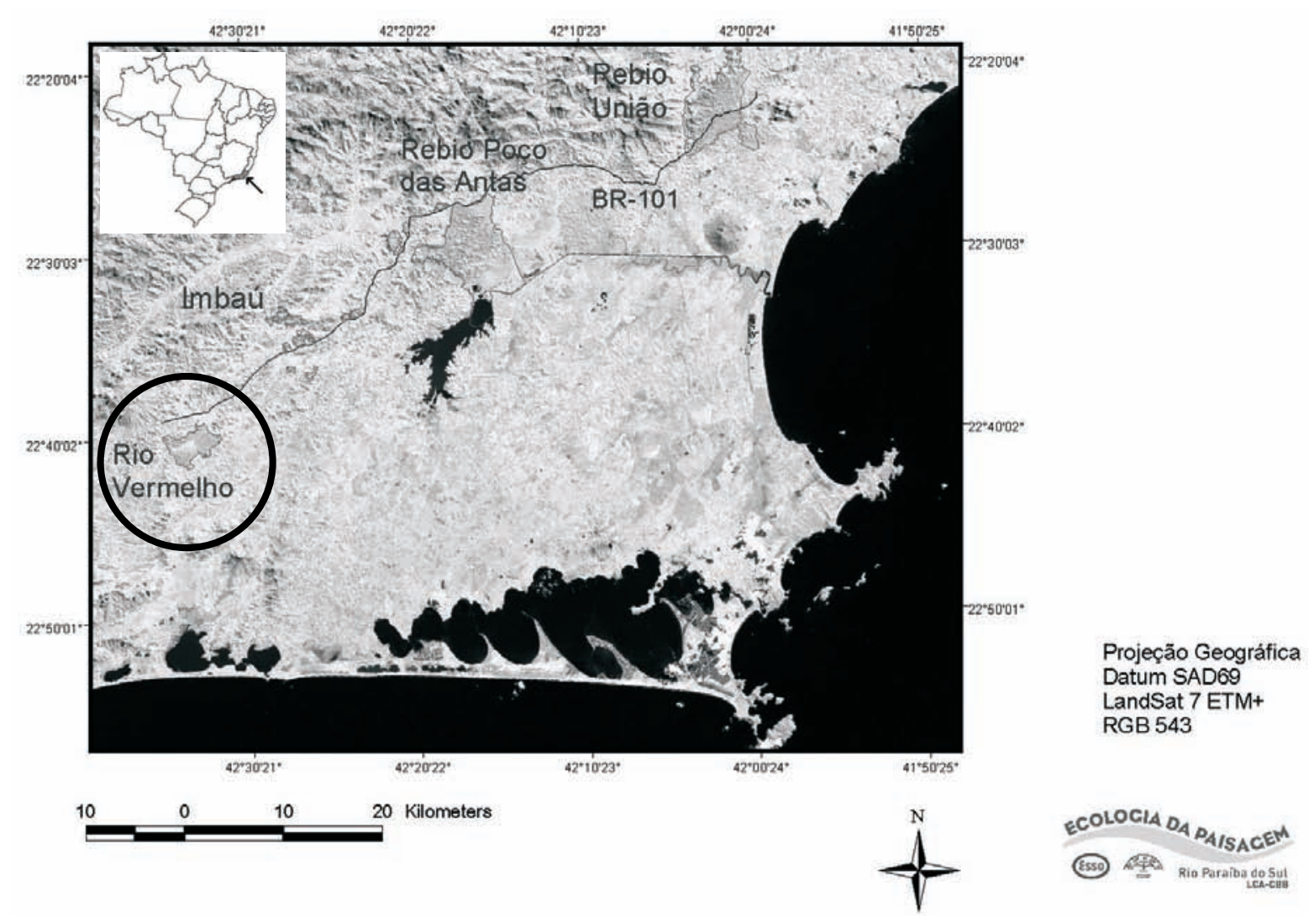

Figura 1 - Localização geográfica da Mata Rio Vermelho no município de Rio Bonito, RJ. Figure 1 - Geographic location of the Rio Vermelho Forest, Rio Bonito, RJ. 
O material botânico coletado foi identificado utilizando literatura especializada e, posteriormente, comparação aos espécimes depositados no herbário do Instituto de Pesquisas Jardim Botânico do Rio de Janeiro (RB) e no acervo de referência da flora regional, na coleção do Herbário do Centro de Biociências e Biotecnologia (UENF). Quando necessário, houve a solicitação de identificação por especialistas. Os espécimes-testemunho foram incorporados na coleção do Herbário do Centro de Biociências e Biotecnologia (UENF), e duplicatas dos materiais férteis encontramse incluídas no Herbário RB. A classificação das famílias seguiu o sistema de Cronquist (1981), exceto para a família Leguminosae, que permaneceu com as subfamílias Caesalpinioideae, Mimosoideae e Papilionoideae. Os binômios das espécies foram atualizados por meio de "software" Royal Botanic Garden of Kew (1993) ou por literatura especializada.

Os parâmetros fitossociológicos foram calculados através do programa FITOPAC 1 (SHEPHERD, 1994), a saber: área basal total e individual (ABt e ABi), densidade relativa (DR), dominância relativa (DoR) e valor de cobertura (VC) (MUELLER-DUMBOIS e ELLENBERG, 1974). O índice de eqüidade (J) e o índice de diversidade de Shannon-Wiener ( $H^{\prime}$ ) foram calculados segundo Brower e Zar (1984).

As espécies levantadas foram classificadas de acordo com suas características sucessionais em pioneiras, secundárias iniciais e secundárias tardias, segundo proposto por Gandolfi et al. (1995) e com base em bibliografia especializada e observações de campo dos autores. Os dados estruturais foram comparados com os obtidos por Rodrigues (2004) para um trecho preservado da Reserva Biológica União, que apresenta a mesma fitofisionomia (Floresta Ombrófila Densa Submontana) e área amostral idêntica de 0,4 ha. A distância entre os dois remanescentes é de cerca de 100 km (Figura 1).

\section{RESULTADOS E DISCUSSÃO}

\subsection{Estrutura}

Foram registrados 776 indivíduos (DAP $\geq 5,0 \mathrm{~cm}$ ), sendo 698 vivos e 78 mortos em pé, compondo uma área basal total de $11,6 \mathrm{~m}^{2}$. As árvores do dossel variaram de altura entre 7-17 m, com algumas emergentes atingindo até $30 \mathrm{~m}$. O DAP médio foi de $12,0 \pm 8,3 \mathrm{~cm}$, com valor máximo de 60,9 cm (Quadro 1). Os valores de área basal e número de árvores de grande porte foram muito inferiores ao da mata preservada da Reserva Biológica União (Quadro 1). Baixos valores de área basal do estrato arbóreo foram relatados por Silva e Nascimento (2001) como sendo reflexo do grau de perturbação antrópica na área, seguindo os padrões de degradação observados nas matas do domínio atlântico, como corte seletivo e retirada de lenha (GIULIETTI e FORERO, 1990). De fato, outras matas consideradas bem preservadas no Estado do Rio de Janeiro, como a floresta de encosta do Imbé, RJ (MORENO et al., 2003), chegam a apresentar valores de área basal para o estrato arbóreo acima dos $40 \mathrm{~m}^{2} \cdot \mathrm{ha}^{-1}$.

Quadro 1 - Comparação estrutural entre a Mata Rio Vermelho e a mata preservada da Reserva Biológica União Table 1 -Structural comparison between Rio Vermelho forest and the preserved forest of União Biological Reserve

\begin{tabular}{|c|c|c|c|}
\hline Parâmetros estruturais & & elho & União \\
\hline Área total amostrada & 0,4 ha & 0,4 ha & 0,4 ha \\
\hline Inclusão diamétrica & $\geq 5,0 \mathrm{~cm}$ & $\geq 10,0 \mathrm{~cm}$ & $\geq 10,0 \mathrm{~cm}$ \\
\hline Número de indivíduos & 698 & 293 & 240 \\
\hline Densidade $\left(\mathrm{ha}^{-1}\right)$ & 1745 & 733 & 600 \\
\hline Número de espécies & 106 & 76 & 117 \\
\hline Área basal total $\left(\mathrm{m}^{2}\right)$ & 11,6 & 9,9 & 16,5 \\
\hline Área basal $\left(\mathrm{m}^{2} \cdot \mathrm{ha}^{-1}\right)$ & 29,0 & 24,8 & 41,3 \\
\hline Altura das árvores (m) & $3,0-13,0$ & $7,0-17,0$ & $10,0-20,0$ \\
\hline Altura máxima das árvores (m) & 30,0 & 30,0 & 37,0 \\
\hline Diâmetro arbóreo médio $(\mathrm{cm})$ & $12,0 \pm 8,3$ & $18,6 \pm 9,3$ & $20,0 \pm 12,0$ \\
\hline Diâmetro arbóreo máximo $(\mathrm{cm})$ & 60,9 & 60,9 & 155,0 \\
\hline Árvores mortas em pé & $78(10,1 \%)$ & $41(12,3 \%)$ & $14(5,5 \%)$ \\
\hline Árvores com lianas & $299(57,2 \%)$ & $199(67,9 \%)$ & $75(31,2 \%)$ \\
\hline Árvores com troncos múltiplos & $58(8,3 \%)$ & $26(8,9 \%)$ & $4(1,7 \%)$ \\
\hline Árvores grandes ( $\geq 30 \mathrm{~cm}$ DAP) & $34(4,9 \%)$ & $34(11,6 \%)$ & $55(22,9 \%)$ \\
\hline Diversidade de espécies (H') & 3,91 & 3,83 & 4,40 \\
\hline Eqüidade $(\mathrm{J})$ & 0,84 & 0,88 & 0,87 \\
\hline
\end{tabular}

R. Árvore, Viçosa-MG, v.31, n.4, p.717-730, 2007 
As árvores vivas amostradas apresentaram padrão de distribuição diamétrica em forma de J-reverso, com ocorrência de maior densidade de árvores na classe de 5-10 cm de DAP e poucos indivíduos atingindo DAP superiores a $20 \mathrm{~cm}$ (Figura 2). O fato de as árvores vivas apresentarem padrão de distribuição em forma de J-reverso não indica, necessariamente, a ausência de problemas de regeneração, sendo necessária uma análise mais detalhada, em nível específico, e com maior grupo de espécies para a confirmação de tal fato.

A ausência de árvores de grande diâmetro pode estar relacionada ao corte seletivo na área e à mortalidade ocasionada pelo efeito de borda, como já observado em outros fragmentos de florestas tropicais (LAURANCE et al., 2000; SCARIOT et al., 2003). Paralelamente, o aumento no número de árvores pequenas (DAP 10$30 \mathrm{~cm}$ ) pode ter sido favorecido pela retirada dos indivíduos do dossel, facilitando o crescimento de juvenis anteriormente estabelecidos e sugerindo que no local ocorreu alteração nos processos sucessionais internos e nos padrões estruturais (LAURANCE et al., 1998).

Um alto número de árvores mortas em pé foi encontrado $(\mathrm{n}=78)$, perfazendo $8,3 \%$ do total de indivíduos amostrados. Grande parte $(83,3 \%)$ dos indivíduos mortos possuía DAP inferior a $20 \mathrm{~cm}$, indicando que a alta mortalidade arbórea não ocorre apenas após a fragmentação, mas persiste por longo período (TABANEZ et al., 1997). Mesmo em relação ao dossel, a Mata Rio Vermelho apresentou 41 indivíduos mortos, contra apenas 14 observados na mata preservada da Reserva Biológica União (Quadro 1). Alta mortalidade arbórea vem sendo apontada como um processo de exclusão característico de florestas fragmentadas, devido às alterações físicas e biológicas potencializadas pelos efeitos de borda (MURCIA, 1995; SCARIOT et al., 2003).

O elevado número de árvores com lianas e indivíduos arbóreos com troncos múltiplos (Quadro 1) aparece como um reflexo dos impactos mais profundos e condições mais estressantes, às quais a mata Rio Vermelho foi submetida. Segundo Putz (1984), as florestas tropicais são ricas em lianas, que exercem, por exemplo, papel importante na alimentação de animais frugívoros durante épocas em que as árvores não frutificam. Entretanto, determinadas espécies de lianas podem interferir no desenvolvimento e sobrevivência das árvores, através da competição por luz, do dano direto das partes aéreas, do sombreamento das plântulas e provocando excesso de peso sobre a copa, causando a queda múltipla de árvores em fortes ventanias e, conseqüentemente, potencializando a mortalidade arbórea. Já o elevado número de troncos múltiplos aparece como um reflexo da queda de árvores e, principalmente, do corte seletivo no local. Portanto, esses resultados indicam que a mata estudada apresenta características estruturais próprias de áreas secundárias, devido ao grau de perturbação (corte seletivo, fragmentação e efeito de borda), ao qual tem sido submetida.

\subsection{Florística e fitossociologia}

Os 698 indivíduos vivos amostrados foram distribuídos em 106 espécies pertencentes a 77 gêneros e 32 famílias. Desses, 675 indivíduos (96,7\% do total) foram identificados em nível de gênero e, ou, espécie, e 18 indivíduos $(2,6 \%)$ foram identificados em nível de família e cinco $(0,7 \%)$ ainda indeterminados. Dentre as espécies, 41 (38,7\% do total) ocorreram com apenas um indivíduo (Quadro 2).

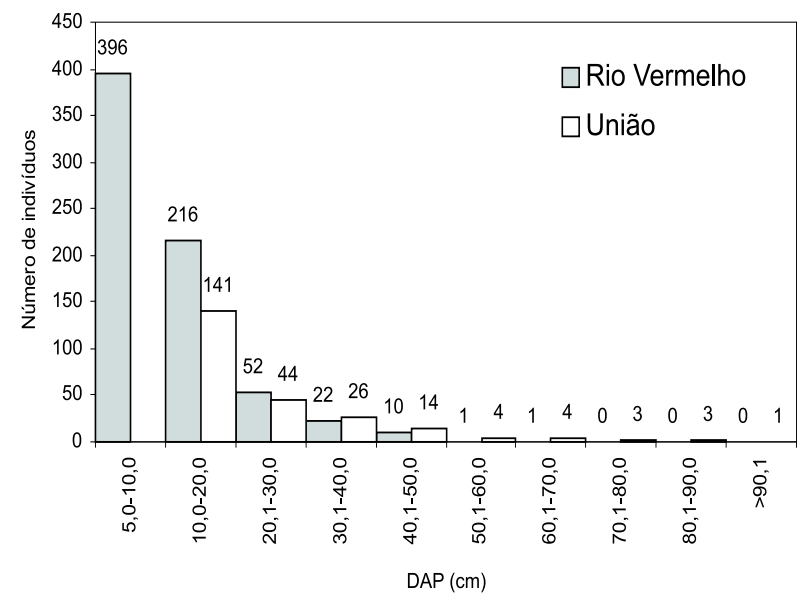

Figura 2 - Distribuição diamétrica dos indivíduos arbóreos amostrados na Mata Rio Vermelho $(\mathrm{n}=698$; DAP $\geq 5,0 \mathrm{~cm}$; área amostral $=0,4 \mathrm{ha}) \mathrm{e}$ comparação com a mata preservada na Reserva Biológica União $(\mathrm{n}=240 ; \mathrm{DAP} \geq 10,0 \mathrm{~cm}$; área amostral $=0,4 \mathrm{ha})$

Figure 2-Diameter distribution of the trees sampled in the Rio Vermelho Forest $(n=698 ; D B H \geq 5$ $\mathrm{cm}$; sampling area $=0.4 \mathrm{ha}$ ) and comparison with the preserved forest of the União Biological Reserve $(n=240 ; D B H \geq 10,0 \mathrm{~cm}$; sampling area $=0.4 \mathrm{ha})$.

R. Árvore, Viçosa-MG, v.31, n.4, p.717-730, 2007 
Quadro 2-Espécies arbóreas amostradas na Mata Rio Vermelho, Rio Bonito, RJ, e respectivos grupos ecológicos $(\mathrm{Pi}=$ pioneira; $\mathrm{Si}=$ secundária inicial $; \mathrm{St}=$ secundária tardia; e $\mathrm{Nc}=$ não classificada)

Table 2 - Tree species recorded in the Rio Vermelho forest, Rio Bonito-RJ, and their ecological groups $(\mathrm{Pi}=$ pionner; $S i=$ secondary initial; $S t=$ late secondary; $n c=$ non-classified)

\begin{tabular}{|c|c|}
\hline Família/Espécie & $\mathrm{CS}$ \\
\hline \multicolumn{2}{|l|}{ ANACARDIACEAE } \\
\hline Astronium graveolens Jacq. & $\mathrm{Si}$ \\
\hline Tapirira guianensis Aubl. & $\mathrm{Si}$ \\
\hline \multicolumn{2}{|l|}{ ANNONACEAE } \\
\hline Guatteria candolleana Schltdl. & $\mathrm{Si}$ \\
\hline Rollinia laurifolia Schltdl. & $\mathrm{Si}$ \\
\hline Xylopia sericea A. St.-Hil. & $\mathrm{Pi}$ \\
\hline \multicolumn{2}{|l|}{ APOCYNACEAE } \\
\hline Geissospermum leave (Vell.) Miers & $\mathrm{St}$ \\
\hline \multicolumn{2}{|l|}{ Himatanthus lancifolius (Müll. Arg.) Woodson } \\
\hline \multicolumn{2}{|l|}{ Tabernaemontana catharinensis A. DC. } \\
\hline Tabernaemontana laeta Mart. & $\mathrm{St}$ \\
\hline \multicolumn{2}{|l|}{ ARECACEAE } \\
\hline Astrocaryum aculeatissimum (Schott) Burret & $\mathrm{Pi}$ \\
\hline \multicolumn{2}{|l|}{ ASTERACEAE } \\
\hline Gochnatia polymorpha (Less.) Cabrera & $\mathrm{Pi}$ \\
\hline Vernonanthura discolor (Less.) H. Rob. & $\mathrm{Pi}$ \\
\hline \multicolumn{2}{|l|}{ BIGNONIACEAE } \\
\hline Cybistax antisyphilitica (Mart.) Mart. & $\mathrm{Pi}$ \\
\hline Jacaranda bracteata Bureau \& K. Schum. & $\mathrm{Si}$ \\
\hline Sparattosperma leucanthum (Vell.) K. Schum. & $\mathrm{Pi}$ \\
\hline Tabebuia chrysotricha (Mart. ex DC.) Standl. & $\mathrm{St}$ \\
\hline Tabebuia heptaphylla (Vell.) Toledo & $\mathrm{St}$ \\
\hline Tabebuia serratifolia (Vahl) G. Nicholson & $\mathrm{St}$ \\
\hline \multicolumn{2}{|l|}{ BOMBACACEAE } \\
\hline Eriotheca pentaphylla (Vell.) A. Robyns. & $\mathrm{St}$ \\
\hline Pseudobombax grandiflorum (Cav.) A. Robyns & $\mathrm{St}$ \\
\hline \multicolumn{2}{|l|}{ CARICACEAE } \\
\hline Jacaratia spinosa (Aubl.) A. DC. & $\mathrm{St}$ \\
\hline \multicolumn{2}{|l|}{ CLUSIACEAE } \\
\hline Garcinia brasiliensis Mart. & $\mathrm{Nc}$ \\
\hline Tovomitopsis paniculata (Spreng.) & $\mathrm{Nc}$ \\
\hline \multicolumn{2}{|l|}{ Planch. \& Triana } \\
\hline Vismia guianensis (Aubl.) Pers. & $\mathrm{Si}$ \\
\hline \multicolumn{2}{|l|}{ ERYTHROXYLACEAE } \\
\hline Erythroxylum pulchrum A. St.-Hil. & $\mathrm{St}$ \\
\hline \multicolumn{2}{|l|}{ EUPHORBIACEAE } \\
\hline Alchornea glandulosa Poepp. & $\mathrm{Si}$ \\
\hline Aparisthmium cordatum (Juss.) Baill. & $\mathrm{Si}$ \\
\hline \multicolumn{2}{|l|}{ Croton floribundus Spreng. } \\
\hline \multicolumn{2}{|l|}{ Mabea fistulifera Mart. } \\
\hline \multirow{2}{*}{$\begin{array}{l}\text { Pera glabrata (Schott) Poepp. ex Baill. } \\
\text { Pera leandri Baill. }\end{array}$} & $\mathrm{Si}$ \\
\hline & $\mathrm{St}$ \\
\hline Sapium glandulatum (Vell.) Pax & $\mathrm{Si}$ \\
\hline
\end{tabular}

Quadro 2 - Cont

Table 2-Cont.

Família/Espécie CS

FLACOURTIACEAE

Casearia arborea (Rich.) Urb. $\quad \mathrm{Si}$

Casearia sylvestris $\mathrm{SW}$. $\mathrm{Si}$

LACISTEMACEAE

Lacistema pubescens Mart. St

LAURACEAE

Aiouea saligna Meisn.

Beilschmiedia fluminensis Kosterm.

Nectandra oppositifolia Nees $\quad \mathrm{Si}$

Ocotea glaziovii Mez St

Ocotea odorifera (Vell.) Rohwer St

Ocotea puberula (Rich.) Nees St

Ocotea schottii (Meisn.) Mez St

Persea americana Mill. Nc

LECYTHIDACEAE

Couratari pyramidata (Vell.) R. Knuth St

LEGUMINOSAE

CAESALPINIOIDEAE

Apuleia leiocarpa (Vogel) J.F. Macbr. Si

Chamaecrista ensiformis (Vell.) $\quad \mathrm{Si}$

Melanoxylon brauna Schott St

MIMOSOIDEAE

Albizia polycephala (Benth.) Killip ex Record $\quad \mathrm{Si}$

Inga cilindrica (Vell.) Mart. St

Piptadenia gonoacantha (Mart.) J.F. Macbr. Nc

Plathymenia foliolosa Benth. St

Pseudopiptadenia contorta (DC.) $\mathrm{Pi}$

G.P. Lewis \& M.P. Lima

PAPILIONOIDEAE

Andira anthelmia (Vell.) J.F. Macbr. St

Andira fraxinifolia Benth. St

Dalbergia nigra (Vell.) Allemão ex Benth. St

Machaerium brasiliense Vogel Si

Swartzia simplex (Sw.) Spreng. St

MALPIGHIACEAE

Byrsonima $\mathrm{sp} 1$

MELASTOMATACEAE

Miconia cinnamomifolia (DC.) Naudin $\quad \mathrm{Pi}$

Miconia sp1 $\quad \mathrm{Pi}$

Tibouchina mutabilis Cogn. $\quad \mathrm{Pi}$

MELIACEAE
Cabralea canjerana (Vell.) Mart.

Cedrela fissilis Vell. $\quad$ St

Cedrela odorata $\mathrm{L}$. $\quad \mathrm{St}$

Guarea guidonia (L.) Sleumer $\quad \mathrm{Si}$

Guarea macrophylla Vahl St

Trichilia lepidota Mart. St

Trichilia silvatica C. DC. $\quad$ St

MONIMIACEAE

Mollinedia puberula Perkins $\quad \mathrm{Si}$

Siparuna guianensis Aubl. $-\ldots-\ldots-\ldots$

Continua ...

Continued...

R. Árvore, Viçosa-MG, v.31, n.4, p.717-730, 2007 
Quadro 2-Cont.

Table 2-Cont.

Família/Espécie CS

MORACEAE

Brosimum guianense (Aubl.) Huber

Ficus adhatodifolia Schott ex Spreng.

Helicostylis tomentosa (Poepp. \& Endl.) Rusby

Sorocea hilarii Gaudich.

MYRISTICACEAE

Virola gardneri (A. DC.) Warb. St

MYRSINACEAE

Myrsine coriacea (Sw.) R. Vr. ex Roem. \& Schult. St MYRTACEAE

Eugenia olivacea O.Berg

Eugenia velutiflora Kiaersk.

Myrcia fallax (Rich.) DC.

Myrcia rostrata DC.

Myrtaceae sp1

Myrtaceae sp2

Syzygium jambos (L.) Alston

NYCTAGINACEAE

Guapira nitida (Mart. ex Schmidt) Lundell

Guapira opposita (Vell.) Reitz

RUBIACEAE

Bathysa mendoncaei K. Schum.

Bathysa meridionalis L.B.Sm. \& Downs

Coussarea nodosa (Benth.) Müll. Arg.

Psychotria vellosiana Benth.

SAPINDACEAE

Cupania oblongifolia Mart.

Cupania racemosa (Vell.) Radlk.

SAPOTACEAE

Chrysophyllum flexuosum Mart.

Ecclinusa ramiflora Mart.

Pouteria caimito (Ruiz \& Pav.) Radlk.

SIMAROUBACEAE

Simarouba amara Aubl.

SOLANACEAE

Aureliana fasciculata (Vell.) Sendtn.

Solanum sp 1

Solanum swartzianum Roem. \& Schult.

TILIACEAE

Luehea divaricata Mart.

Luehea grandiflora Mart. \& Zucc.

INDETERMINADA

Indeterminada $\mathrm{sp} 1$

Indeterminada $\mathrm{sp} 2$

Indeterminada $\mathrm{sp} 3$

Indeterminada sp4

Indeterminada sp5

Indeterminada sp6

\begin{tabular}{cc} 
& $\mathrm{Nc}$ \\
& $\mathrm{Nc}$ \\
& $\mathrm{Nc}$ \\
& $\mathrm{Nc}$ \\
& $\mathrm{Nc}$ \\
& $\mathrm{Nc}$ \\
\hline Total famílias -32 & \\
Total gêneros -77 & \\
Total espécies -106 & \\
\hline
\end{tabular}

Nove famílias (28,1\% do total) ocorreram com uma única espécie. As famílias com maior riqueza de espécies foram Leguminosae (13 espécies), Lauraceae (8), Euphorbiaceae (7), Meliaceae (7), Myrtaceae (7) e Bignoniaceae (6), e as de maior número de indivíduos foram Monimiaceae (88 indivíduos), Leguminosae (79), Euphorbiaceae (63), Meliaceae (47), Moraceae (45) e Nyctaginaceae (42). Dentre os gêneros, Ocotea e Tabebuia foram os que apresentaram o maior número de espécies, com quatro e três, respectivamente.

Segundo Oliveira-Filho e Fontes (2000), as famílias Leguminosae, Lauraceae e Myrtaceae são as que geralmente ocorrem com os maiores números de espécies ao longo da Mata Atlântica costeira brasileira. Gentry (1982) considerou que a família Leguminosae encontrase entre as mais ricas em espécies nas matas neotropicais de terras baixas, e Lima (2000) ressaltou o aumento da expressividade dessa família nas florestas submontanas do Estado do Rio de Janeiro, relacionando a um maior estoque de suas espécies nessa faixa altitudinal.

Monimiaceae, Sapindaceae e Nyctaginaceae destacaram-se pela importância de uma ou duas espécies (Monimiaceae $=$ Siparuna guianensis, Sapindaceae = Cupania oblongifolia e C. racemosa, Nyctaginaceae = Guapira nitida e G. opposita), fazendo com que exista certa dominância no nível de família, característica que aparenta ser comum em florestas tropicais (RICHARDS, 1981). Entretanto, as demais famílias como Leguminosae, Euphorbiaceae e Meliaceae não apresentam essa característica tão clara, destacando-se, principalmente por sua riqueza de espécies relativamente alta, um padrão comum a outras florestas da costa brasileira (OLIVEIRA-FILHO e FONTES, 2000).

A espécie mais representativa nos parâmetros fitossociológicos foi Siparuna guianensis, pela sua elevada abundância. Outras espécies de destaque foram Apuleia leiocarpa, Cupania oblongifolia, Machaerium brasiliense, Xylopia sericea, Simarouba amara, Helicostylis tomentosa, Guapira nitida, Lacistema pubescens e Psychotria vellosiana (Quadro 3). Essas 10 espécies em conjunto representaram $43 \%$ do VC total e $40 \%$ do total de indivíduos amostrados. Algumas dessas espécies, típicas de sub-bosque, apresentaram baixos valores médios de DAP, mas se destacaram pelo elevado número de indivíduos, como Helicostilis tomentosa, Guapira nitida, Lacistema pubescens, Psychotria vellosiana, Pera glabrata, Guapira opposita

R. Árvore, Viçosa-MG, v.31, n.4, p.717-730, 2007 
CARVALHO, F.A. et al.

e Guarea guidonia. Outras características de dossel e, ou, emergentes apresentam baixo número de indivíduos, mas elevado DAP médio, como no caso das já citadas
Simarouba amara, Apuleia leiocarpa e Machaerium brasiliense, Pera leandri, Plathymenia foliolosa e Pseudopiptadenia contorta (Quadro 3).

Quadro 3 - Parâmetros fitossociológicos das espécies arbóreas amostradas na Mata Rio Vermelho, Rio Bonito, RJ. Ni = número de indivíduos, $\mathrm{DR}=$ densidade relativa, $\mathrm{DoR}=$ dominância relativa, $\mathrm{VC}=$ valor de cobertura e $\mathrm{AB}=$ área basal $\left(\mathrm{m}^{2}\right)$

Table 3 - Phytosociological parameters of tree species recorded in the Rio Vermelho Forest, Rio Bonito-RJ. Ni $=n u m b e r$ of individuals; $D R=$ relative density; DoR = relative dominance $; C=$ cover value; $A B=$ basal area $\left(\mathrm{m}^{2}\right)$

\begin{tabular}{|c|c|c|c|c|c|c|}
\hline & Espécie & $\mathrm{Ni}$ & DR & DoR & $\mathrm{VC}$ & $\mathrm{AB}$ \\
\hline 1 & Siparuna guianensis & 86 & 12,32 & 3,06 & 15,38 & 0,356 \\
\hline 2 & Apuleia leiocarpa & 13 & 1,86 & 8,15 & 10,01 & 0,948 \\
\hline 3 & Cupania oblongifolia & 25 & 3,58 & 6,39 & 9,98 & 0,744 \\
\hline 4 & Machaerium brasiliense & 15 & 2,15 & 6,76 & 8,91 & 0,788 \\
\hline 5 & Helicostylis tomentosa & 34 & 4,87 & 3,55 & 8,42 & 0,413 \\
\hline 6 & Guapira nitida & 21 & 3,01 & 4,60 & 7,61 & 0,536 \\
\hline 7 & Lacistema pubescens & 35 & 5,01 & 1,90 & 6,92 & 0,222 \\
\hline 8 & Xylopia sericea & 20 & 2,87 & 3,70 & 6,57 & 0,431 \\
\hline 9 & Psychotria vellosiana & 26 & 3,72 & 2,82 & 6,55 & 0,328 \\
\hline 10 & Simarouba amara & 6 & 0,86 & 5,60 & 6,46 & 0,652 \\
\hline 11 & Cabralea canjerana & 19 & 2,72 & 3,38 & 6,11 & 0,394 \\
\hline 12 & Chamaecrista ensiformis & 25 & 3,58 & 1,67 & 5,26 & 0,195 \\
\hline 13 & Pera glabrata & 26 & 3,72 & 0,96 & 4,69 & 0,112 \\
\hline 14 & Pera leandri & 8 & 1,15 & 3,39 & 4,54 & 0,395 \\
\hline 15 & Guapira opposita & 21 & 3,01 & 1,47 & 4,48 & 0,172 \\
\hline 16 & Astrocaryum aculeatissimum & 18 & 2,58 & 1,82 & 4,40 & 0,212 \\
\hline 17 & Guarea guidonia & 20 & 2,87 & 1,26 & 4,12 & 0,146 \\
\hline 18 & Plathymenia foliolosa & 9 & 1,29 & 2,72 & 4,01 & 0,316 \\
\hline 19 & Pseudopiptadenia contorta & 6 & 0,86 & 2,93 & 3,79 & 0341 \\
\hline 20 & Casearia arborea & 12 & 1,72 & 2,02 & 3,74 & 0,235 \\
\hline 21 & Tapirira guianensis & 6 & 0,86 & 2,36 & 3,22 & 0,275 \\
\hline 22 & Myrtaceae sp1 & 17 & 2,44 & 0,69 & 3,13 & 0,080 \\
\hline 23 & Mabea fistulifera & 8 & 1,15 & 1,96 & 3,11 & 0,228 \\
\hline 24 & Cupania racemosa & 10 & 1,43 & 1,43 & 2,87 & 0,167 \\
\hline 25 & Ficus adhatodifolia & 2 & 0,29 & 2,51 & 2,80 & 0,292 \\
\hline 26 & Ocotea glaziovii & 13 & 1,86 & 0,78 & 2,64 & 0,090 \\
\hline 27 & Aparisthmium cordatum & 12 & 1,72 & 0,77 & 2,49 & 0,090 \\
\hline 28 & Byrsonima sp & 4 & 0,57 & 1,74 & 2,32 & 0,203 \\
\hline 29 & Piptadenia gonoacantha & 1 & 0,14 & 1,54 & 1,68 & 0,179 \\
\hline 30 & Cybistax antisyphilitica & 9 & 1,29 & 0,37 & 1,66 & 0,043 \\
\hline 31 & Myrcia rostrata & 8 & 1,15 & 0,37 & 1,51 & 0,043 \\
\hline 32 & Brosimum guianense & 6 & 0,86 & 0,59 & 1,45 & 0,068 \\
\hline 33 & Melanoxylon brauna & 1 & 0,14 & 1,30 & 1,45 & 0,152 \\
\hline 34 & Sparattosperma leucanthum & 4 & 0,57 & 0,78 & 1,36 & 0,091 \\
\hline 35 & Syzygium jambos & 6 & 0,86 & 0,48 & 1,34 & 0,056 \\
\hline 36 & Guarea macrophylla & 4 & 0,57 & 0,76 & 1,34 & 0,089 \\
\hline 37 & Coussarea nodosa & 7 & 1,00 & 0,30 & 1,30 & 0,035 \\
\hline 38 & Croton floribundus & 4 & 0,57 & 0,72 & 1,29 & 0,084 \\
\hline 39 & Astronium graveolens & 5 & 0,72 & 0,48 & 1,20 & 0,056 \\
\hline 40 & Miconia sp1 & 3 & 0,43 & 0,76 & 1,19 & 0,089 \\
\hline 41 & Couratari pyramidata & 6 & 0,86 & 0,32 & 1,18 & 0,037 \\
\hline 42 & Ocotea schottii & 5 & 0,72 & 0,39 & 1,11 & 0,046 \\
\hline
\end{tabular}

R. Árvore, Viçosa-MG, v.31, n.4, p.717-730, 2007 
Quadro 3-Cont.

Table 3 - Cont

\begin{tabular}{|c|c|c|c|c|c|c|}
\hline & Espécie & $\mathrm{Ni}$ & DR & DoR & $\mathrm{VC}$ & $\mathrm{AB}$ \\
\hline 43 & Guatteria candolleana & 5 & 0,72 & 0,36 & 1,08 & 0,042 \\
\hline 44 & Gochnatia polymorpha & 6 & 0,86 & 0,21 & 1,07 & 0,024 \\
\hline 45 & Luehea divaricata & 4 & 0,57 & 0,39 & 0,96 & 0,045 \\
\hline 46 & Rollinia laurifolia & 3 & 0,43 & 0,46 & 0,89 & 0,053 \\
\hline 47 & Cedrela odorata & 1 & 0,14 & 0,73 & 0,87 & 0,084 \\
\hline 48 & Vismia guianensis & 3 & 0,43 & 0,44 & 0,87 & 0,051 \\
\hline 49 & Alchornea glandulosa & 4 & 0,57 & 0,29 & 0,86 & 0,033 \\
\hline 50 & Himatanthus lancifolius & 3 & 0,43 & 0,37 & 0,80 & 0,043 \\
\hline 51 & Myrcia fallax & 4 & 0,57 & 0,21 & 0,78 & 0,024 \\
\hline 52 & Tabernaemontana laeta & 2 & 0,29 & 0,46 & 0,75 & 0,053 \\
\hline 53 & Tabebuia serratifolia & 2 & 0,29 & 0,44 & 0,73 & 0,051 \\
\hline 54 & Ocotea odorifera & 3 & 0,43 & 0,24 & 0,67 & 0,027 \\
\hline 55 & Aiouea saligna & 3 & 0,43 & 0,23 & 0,66 & 0,026 \\
\hline 56 & Sorocea hilarii & 3 & 0,43 & 0,16 & 0,59 & 0,019 \\
\hline 57 & Jacaranda bracteata & 3 & 0,43 & 0,16 & 0,59 & 0,019 \\
\hline 58 & Eugenia olivacea & 3 & 0,43 & 0,16 & 0,59 & 0,018 \\
\hline 59 & Swartzia simplex & 1 & 0,14 & 0,44 & 0,59 & 0,052 \\
\hline 60 & Chrysophyllum flexuosum & 3 & 0,43 & 0,15 & 0,58 & 0,018 \\
\hline 61 & Tibouchina mutabilis & 3 & 0,43 & 0,12 & 0,55 & 0,014 \\
\hline 62 & Geissospermum leave & 1 & 0,14 & 0,37 & 0,52 & 0,044 \\
\hline 63 & Myrsine coriacea & 1 & 0,14 & 0,35 & 0,50 & 0,041 \\
\hline 64 & Andira fraxinifolia & 2 & 0,29 & 0,20 & 0,49 & 0,024 \\
\hline 65 & Tabernaemontana catharinensis & 1 & 0,14 & 0,32 & 0,46 & 0,037 \\
\hline 66 & Indeterminada $\mathrm{sp} 1$ & 1 & 0,14 & 0,32 & 0,46 & 0,037 \\
\hline 67 & Persea americana & 2 & 0,29 & 0,10 & 0,39 & 0,012 \\
\hline 68 & Bathysa mendoncaei & 2 & 0,29 & 0,09 & 0,38 & 0,011 \\
\hline 69 & Inga cilindrica & 2 & 0,29 & 0,08 & 0,37 & 0,001 \\
\hline 70 & Indeterminada $\mathrm{sp} 3$ & 1 & 0,14 & 0,21 & 0,35 & 0,024 \\
\hline 71 & Casearia sylvestris & 2 & 0,29 & 0,07 & 0,35 & 0,008 \\
\hline 72 & Mollinedia puberula & 2 & 0,29 & 0,06 & 0,35 & 0,008 \\
\hline 73 & Bathysa meridionalis & 2 & 0,29 & 0,06 & 0,35 & 0,007 \\
\hline 74 & Eriotheca pentaphylla & 1 & 0,14 & 0,20 & 0,35 & 0,024 \\
\hline 75 & Andira anthelmia & 2 & 0,29 & 0,05 & 0,34 & 0,006 \\
\hline 76 & Pouteria caimito & 1 & 0,14 & 0,18 & 0,33 & 0,021 \\
\hline 77 & Luehea grandiflora & 1 & 0,14 & 0,17 & 0,31 & 0,020 \\
\hline 78 & Albizia polycephala & 1 & 0,14 & 0,14 & 0,28 & 0,016 \\
\hline 79 & Dalbergia nigra & 1 & 0,14 & 0,11 & 0,26 & 0,013 \\
\hline 80 & Jacaratia spinosa & 1 & 0,14 & 0,11 & 0,25 & 0,013 \\
\hline 81 & Solanum swartzianum & 1 & 0,14 & 0,10 & 0,25 & 0,012 \\
\hline 82 & Pseudobombax grandiflorum & 1 & 0,14 & 0,10 & 0,24 & 0,012 \\
\hline 83 & Miconia cinnamomifolia & 1 & 0,14 & 0,09 & 0,23 & 0,011 \\
\hline 84 & Tabebuia chrysotricha & 1 & 0,14 & 0,08 & 0,22 & 0,009 \\
\hline 85 & Cedrela fissilis & 1 & 0,14 & 0,08 & 0,22 & 0,009 \\
\hline 86 & Indeterminada sp4 & 1 & 0,14 & 0,06 & 0,21 & 0,007 \\
\hline 87 & Indeterminada $\mathrm{sp} 2$ & 1 & 0,14 & 0,06 & 0,21 & 0,007 \\
\hline 88 & Vernonanthura discolor & 1 & 0,14 & 0,06 & 0,20 & 0,007 \\
\hline 89 & Virola gardneri & 1 & 0,14 & 0,06 & 0,20 & 0,007 \\
\hline 90 & Aureliana fasciculata & 1 & 0,14 & 0,06 & 0,20 & 0,007 \\
\hline 91 & Ocotea puberula & 1 & 0,14 & 0,05 & 0,20 & 0,006 \\
\hline 92 & Solanum sp1 & 1 & 0,14 & 0,04 & 0,18 & 0,004 \\
\hline 93 & Myrtaceae sp2 & 1 & 0,14 & 0,04 & 0,18 & 0,004 \\
\hline
\end{tabular}

Contin

Continued... 
Quadro 3 - Cont.

Table 3 - Cont.

\begin{tabular}{|c|c|c|c|c|c|c|}
\hline & Espécie & $\mathrm{Ni}$ & DR & DoR & $\mathrm{VC}$ & $\mathrm{AB}$ \\
\hline 94 & Tabebuia heptaphylla & 1 & 0,14 & 0,03 & 0,18 & 0,004 \\
\hline 95 & Indeterminada sp5 & 1 & 0,14 & 0,03 & 0,18 & 0,004 \\
\hline 96 & Ecclinusa ramiflora & 1 & 0,14 & 0,03 & 0,18 & 0,004 \\
\hline 97 & Erythroxylum pulchrum & 1 & 0,14 & 0,03 & 0,17 & 0,003 \\
\hline 98 & Tovomitopsis paniculata & 1 & 0,14 & 0,03 & 0,17 & 0,003 \\
\hline 99 & Indeterminada sp6 & 1 & 0,14 & 0,02 & 0,17 & 0,003 \\
\hline 100 & Trichilia silvatica & 1 & 0,14 & 0,02 & 0,17 & 0,003 \\
\hline 101 & Sapium glandulatum & 1 & 0,14 & 0,02 & 0,16 & 0,002 \\
\hline 102 & Nectandra oppositifolia & 1 & 0,14 & 0,02 & 0,16 & 0,002 \\
\hline 103 & Beilschmiedia fluminensis & 1 & 0,14 & 0,02 & 0,16 & 0,002 \\
\hline 104 & Garcinia brasiliensis & 1 & 0,14 & 0,02 & 0,16 & 0,002 \\
\hline 105 & Eugenia velutiflora & 1 & 0,14 & 0,02 & 0,16 & 0,002 \\
\hline \multirow[t]{2}{*}{106} & Trichilia lepidota & 1 & 0,14 & 0,02 & 0,16 & 0,002 \\
\hline & Total & 698 & 100,0 & 100,0 & 200,0 & 11,642 \\
\hline
\end{tabular}

O domínio de Siparuna guianensis nos parâmetros fitossociológicos, como encontrado na Mata Rio Vermelho, não foi até o momento demonstrado em nenhum fragmento florestal dessa região, apesar de existirem registros do gênero Siparuna como um dos mais importantes em sub-bosques de matas em regeneração secundária. Neves (1999) na Reserva Biológica de Poço das Antas, encontrou Siparuna guianensis como a nona mais importante (VC) no sub-bosque de uma mata com 20 anos de regeneração, e a terceira mais importante no sub-bosque de uma mata com 40 anos de regeneração. Rabelo et al. (2001) registraram Siparuna sp. como uma das espécies mais abundantes no sub-bosque de plantios abandonados de eucalipto na Reserva Biológica União. Já Carvalho (2005) encontrou Siparuna guianensis entre as 10 espécies mais importantes (VC) em cinco fragmentos florestais secundários na região de Imbaú, Silva Jardim, RJ. Assim, é notável a presença do gênero Siparuna no sub-bosque das matas em regeneração na região, indicando sua preferência por áreas secundárias.

As demais espécies que formam o grupo das mais importantes (VC), Apuleia leiocarpa, Cupania oblongifolia, Machaerium brasiliense, Helicostylis tomentosa, Guapira nitida, Lacistema pubescens, Xylopia sericea, Psychotria vellosiana e Simarouba amara, aparecem também entre as de maior destaque em outros levantamentos nessa região (GUEDES-BRUNI, 1998; NEVES, 1999; BORÉM e OLIVEIRA-FILHO, 2002; PESSOA, 2003; RODRIGUES, 2004; CARVALHO, 2005). Apuleia leiocarpa é citada por Neves (1999) como uma emergente característica de dossel, sendo uma típica remanescente nessas matas. Cupania oblongifolia, Helicostylis tomentosa, Xylopia sericea e Simarouba amara têm sido registradas como espécies características de dossel, cujas abundâncias são maiores em áreas secundárias (NEVES, 1999; PESSOA, 2003; CARVALHO, 2005). Já Guapira nitida, Lacistema pubescens e Psychotria velloziana aparecem na maioria dos levantamentos anteriormente mencionados, de preferência em áreas perturbadas, como típicas de sub-bosque, sendo rara a sua ascensão ao dossel.

O grupo ecológico que mais contribuiu para a composição da comunidade arbórea foi o das secundárias iniciais, com 47,7\% do total de indivíduos amostrados, seguido das secundárias tardias $(29,1 \%)$ e pioneiras $(12,1 \%)$. Essa elevada densidade de espécies pertencentes ao grupo das secundárias iniciais é uma característica de matas perturbadas, visto que em florestas tropicais maduras esse grupo tende a ocorrer em baixas densidades (raras) (HUBBEL et al., 1999). Florestas em fases iniciais de regeneração apresentam, geralmente, elevada abundância de espécies pioneiras, que exercem função cicatrizadora, fato também observado em bordas antrópicas (MURCIA, 1995) e clareiras naturais (TABARELLI e MANTOVANI, 1999). Dentre as poucas espécies pioneiras encontradas na mata Rio Vermelho, as mais abundantes foram arbóreas de ciclo de vida longo, como Xylopia sericea, Pseudopiptadenia contorta e Mabea fistulifera. A baixa representatividade de espécies secundárias tardias, a ausência de pioneiras de ciclo de vida curto, e a elevada densidade de espécies secundárias iniciais, principalmente no dossel, são características de florestas tropicais secundárias em 
fases intermediárias de regeneração (GANDOLFI et al., 1995; HUBBEL et al., 1999).

Dentre as 41 espécies que apresentaram apenas um indivíduo, sendo, portanto, consideradas raras segundo o conceito de Martins (1993), duas merecem destaque: Melanoxylon brauna e Dalbergia nigra. Tais espécies têm sido raramente registradas nos levantamentos florísticos realizados na Mata Atlântica do Estado do Rio de Janeiro e encontram-se na lista oficial das espécies arbóreas ameaçadas de extinção da flora brasileira (IBAMA, 1992). Apesar do conceito de espécie rara como sendo aquela representada por apenas um indivíduo na área de estudo (MARTINS, 1993) e dos elevados índices de endemismo da MataAtlântica costeira (MORI et al., 1981), o fato de tais espécies apresentarem apenas um indivíduo, aliado às suas ausências na maioria dos inventários florísticos no Estado, indica redução de suas populações nos remanescentes do Estado do Rio de Janeiro. Esses padrões também indicam que tais espécies possuem grandes chances de experimentar um rápido declínio em termos populacionais, tendendo a extinções locais, em decorrência dos efeitos da fragmentação florestal (TILMAN et al., 1994; SCARIOT et al., 2003). Tais considerações enfatizaram a necessidade de se conservarem remanescentes com certa representatividade florística e de grande importância como bancos genéticos ex situ.

\subsection{Diversidade}

O valor do índice de diversidade de Shannon-Wiener para a comunidade arbórea da mata Rio Vermelho $\left(\mathrm{H}^{\prime}\right.$ = 3,91 nats) encontra-se entre os mais altos em relação aos de outras matas consideradas perturbadas da região ( $\mathrm{H}^{\prime}=$ 3,02 a 4,14 nats, vide (NEVES, 1999; BORÉM e OLIVEIRA-FILHO, 2002; PESSOA, 2003; CARVALHO, 2005). Entretanto, esse valor mostrou-se bem inferior ao encontrado na mata preservada da Reserva Biológica União ( $H^{\prime}=4,40$ nats, Quadro 1), e nas outras matas maduras da região (H'> 4,3 nats, (GUEDES-BRUNI, 1998; MORENO et al., 2003).

Estudos em florestas tropicais vêm indicando que sítios com maior densidade tendem a apresentar comparativamente maior riqueza de espécies (BROKAW e BUSING, 2000). Entretanto, também pode haver maior densidade quando uma ou mais espécies são localmente favorecidas por distúrbios (LAURANCE et al., 1998). Neste estudo, as 10 espécies mais abundantes foram responsáveis por mais de $50 \%$ do total de indivíduos amostrados, acarretando redução na diversidade em comparação com a mata preservada. Nesse contexto, fatores como a redução da área, mudança na forma e os efeitos de borda proporcionados pela fragmentação florestal, associados à retirada seletiva de madeira, proporcionam redução na variabilidade genética em populações limitadas, e a formação de microclimas que favorecem o estabelecimento de espécies oportunistas, levando a um declínio na riqueza e, consequientemente, na diversidade dos fragmentos florestais (TURNER e CORLETT, 1996).

\section{CONCLUSÕES}

Numa visão geral, os resultados florísticosestruturais (ex. baixos valores de área basal e de densidade de árvores de grande porte, altos valores de árvores mortas, árvores com lianas em suas copas e de espécies de grupos sucessionais iniciais) são indicativo de que a mata estudada encontra-se perturbada e em franco processo de regeneração secundária. Ainda assim, esse remanescente se mantém detentor de considerável riqueza e diversidade florística, apresentando algumas espécies arbóreas da lista oficial de espécies da flora brasileira ameaçadas de extinção. Dada a representatividade desse remanescente para a manutenção da flora e fauna local, medidas concretas visando à sua preservação devem ser implementadas, como a sua transformação em uma Unidade de Conservação e a implementação de práticas de manejo florestal visando à sua recuperação. Recomenda-se, ainda, que sejam desenvolvidos estudos sobre a estrutura e dinâmica de suas populações arbóreas, especialmente aquelas ameaçadas, como Melanoxylon brauna e Dalbergia nigra.

\section{AGRADECIMENTOS}

Aos pesquisadores do Jardim Botânico do Rio de Janeiro Alexandre Quinet, Carine Pinto-Quinet, Cláudia Magalhães Vieira, Cyl Farney de Sá, Massime Giusepp Bovini e Haroldo Cavalcanti Lima, pelo auxílio na identificação do material coletado; aos proprietários da Fazenda Rio Vermelho, por permitirem o livre acesso à área de estudo; a Heuzenil S. Cordeiro e Márcio M. Morais, pelo auxílio nos trabalhos de campo; a Fernando Carvalho e Silva, pela confecção do mapa; ao FNMA/ MMA, pelo apoio financeiro; à Associação Mico Leão Dourado, LCA/CBB/UENF e ao IBAMA, pelo apoio logístico; e a CAPES, pela concessão da bolsa de mestrado ao primeiro autor.

R. Árvore, Viçosa-MG, v.31, n.4, p.717-730, 2007 


\section{REFERÊNCIAS}

BORÉM, R. A. T.; OLIVEIRA-FILHO, A. T. Fitossociologia do estrato arbóreo em uma topossequência alterada de Mata Atlântica, no município de Silva Jardim-RJ, Brasil. Revista Árvore, v.26, n.4, p.727-742, 2002.

BRASIL. Ministério da Agricultura. Avaliação e ações prioritárias para a conservação da biodiversidade da Mata Atlântica e Campos Sulinos. Brasília: 2000. 322p.

BROKAW, N.; BUSING, R. T. Niche versus chance and tree diversity in forest gaps. Trends in Ecology and Evolution, v.15, p.183-188, 2000.

BROWER, J. E.; ZAR, J. H. Field and laboratory methods for general ecology. 2.ed. Iowa: W.C. Brown Company Publishers, 1984. 226p.

CARVALHO, F. A. et al. A importância dos remanescentes florestais da Mata Atlântica da baixada costeira fluminense para a conservação da biodiversidade na APA da Bacia do Rio São João/ Mico-Leão-Dourado, RJ. In: CONGRESSO BRASILEIRO DE UNIDADES DE CONSERVAÇÃO, 4., 2004, Curitiba. Anais... Curitiba: Fundação O Boticário de Proteção a Natureza, 2004. p.106-113.

CARVALHO, F. A. Efeitos da fragmentação florestal na florística e estrutura de fragmentos de Mata Atlântica submontana na região de Imbaú, município de Silva Jardim, RJ. 2005. 124f. Dissertação (Mestrado em Biociências e Biotecnologia) - Universidade Estadual do Norte Fluminense, Campos dos Goytacazes, 2005.

CAUSTON, D. R. An introduction to vegetation analysis, principles, practice and interpretation. London: Unwin, Hyman, 1988. 342p.

CRONQUIST, A. An integrated system of classification of flowering plants. New York: Columbia University Press, 1981. 555p.

DEAN, W. A ferro e fogo - a história e a devastação da Mata Atlântica brasileira. São Paulo: Companhia das Letras, 1996. 484p.

R. Árvore, Viçosa-MG, v.31, n.4, p.717-730, 2007
FUNDAÇÃO SOS MATA ATLÂNTICA. Atlas

da evolução dos remanescentes

florestais e ecossistemas associados no domínio da Mata Atlântica no período 1990-1995. São Paulo: 2002.

GANDOLFI, S.; LEITÃO-FILHO, H. F.; BEZERRA, C. L. F. Levantamento florístico e caráter sucessional das espécies arbustivo-arbóreas de uma floresta mesófila semidecídua no município de Guarulhos, SP. Revista Brasileira de Biologia, v.55, p.753-767, 1995.

GENTRY, A. Neotropical floristic diversity: phytogeografical connections between Central and South America, pleistocene climatic fluctuations or an accident of the Andean orogeny? Annals of Missouri Botanical Garden, v.69, p.557-593, 1982.

GIULIETTI, A. M.; FORERO, H. Diversidade taxonômica e padrões de distribuição das angiospermas brasileiras. Acta Botanica Brasilica, v.4, p.3-9, 1990.

GUEDES-BRUNI, R. R. Composição, estrutura e similaridade florística de dossel em seis unidades de Mata Atlântica no Rio de Janeiro. 1998. $231 \mathrm{f}$. Tese (Doutorado em Ecologia) - Universidade de São Paulo, São Paulo, 1998.

HUBBEL, S. P. et al. Light gaps disturbance, recruitment limitations and tree diversity in a neotropical forest. Science, v.283, p.554-557, 1999.

IBAMA. Lista Oficial das Espécies da Flora Brasileira Ameaçadas de Extinção. Diário Oficial, Portaria 006/92-N de 15 de Janeiro de 1992. 1992.

LAURANCE, W. F. et al. Effects of forest fragmentation on recruitment patterns in Amazonian tree communities. Conservation Biology, v. 12, p.460-464, 1998.

LAURANCE, W. F. et al. Rainforest fragmentation kills big trees. Nature, v.404, p.836, 2000.

LIMA, H. C. Leguminosas arbóreas da Mata Atlântica. 2000. 156f. Tese (Doutorado em Ecologia) - Universidade Federal do Rio de Janeiro, Rio de Janeiro, 2000. 
MARTINS, F. R. Estrutura de uma

floresta mesófila. Campinas: Universidade Estadual de Campinas, 1993. 246p.

MORENO, M. R.; NASCIMENTO, M. T.; KURTZ, B. C. Estrutura e composição florística do estrato arbóreo em duas zonas altitudinais na Mata Atlântica de encosta da região do Imbé, RJ. Acta Botanica Brasilica, v.17, p.371-386, 2003.

MORI, S. A.; BOOM, B. M.; PRANCE, G. T. Distribuition of eastern Brazilian costal forest tree species. Brittonia, v.33, p.233-245, 1981.

MUELLER-DUMBOIS, D.; ELLENBERG, H. Aims and methods of vegetation ecology. New York: John Willey \& Sons Press, 1974. 574p.

MURCIA, C. Edge effects in fragmented forests: implications for conservation. Trends in Ecology and Evolution, v.10, p.58-62, 1995.

NEVES, G. M. S. Florística e estrutura da comunidade arbustivo-arbórea em dois remanescentes de Floresta Atlântica secundária - Reserva Biológica de Poço das Antas, Silva Jardim, RJ. 1999. 115f. Dissertação (Mestrado em Ciências Biológicas) - Universidade Federal do Rio de Janeiro, Rio de Janeiro, 1999.

OLIVEIRA-FILHO, A. T.; FONTES, M. A. L. Patterns of floristic differentiation amog Atlantic Forests in southeastern Brazil and influence of climate. Biotropica, v.32, p.793-810, 2000.

PESSOA, S. V. A. Aspectos da fragmentação em remanescentes florestais da planície costeira do estado do Rio de Janeiro. 2003. 112f. Dissertação (Mestrado em Ciências Florestais) Universidade Federal Rural do Rio de Janeiro, Rio de Janeiro, 2003.

PRIMO, P. B. S.; VÖLKER, C. M. Bacias hidrográficas dos Rios São João e das Ostras - águas, terras e conservação ambiental. Rio de Janeiro: CILSJ, 2003. 115p.

PUTZ, F. E. The natural history of lianas on Barro Colorado Island, Panama. Ecology, v.65, p.1713-1724, 1984.
RABELO, G. R.; CARVALHO, F. A.; NASCIMENTO, M. T. Abordagem preliminar sobre a florística e a estrutura em plantios de eucaliptos de diferentes idades na REBIO União-RJ. In: CONGRESSO NACIONAL DE BOTÂNICA, 52., 2001, João Pessoa.

Resumos... João Pessoa: Sociedade Brasileira de Bôtanica, 2001.p.231.

RICHARDS, P. W. The tropical rain forest: an ecological study. 2.ed. Cambridge: Cambridge University Press, 1981. 262p.

RODRIGUES, P. J. F. P. A vegetação da Reserva Biológica União e os efeitos de borda na Mata Atlântica fragmentada. 2004. 136f. Tese (Doutorado em Biociências e Biotecnologia) - Universidade Estadual do Norte Fluminense, Campos dos Goytacazes, 2004.

ROYAL BOTANICAL GARDEN. Index Kewensis on compact disc - manual. Oxford: Oxford University Press, 1993.

SCARIOT, A. et al. Efeitos da fragmentação sobre a biodiversidade: vegetação e flora. In RAMBALDI, D. M.; OLIVEIRA, D. A. S. Fragmentação de ecossistemas: causas, efeitos sobre a biodiversidade e recomendações de políticas públicas. Brasília: MMA/SBF, 2003. 510p.

SHEPHERD, G. J. FITOPAC 1: Manual do usuário. Campinas: Universidade Estadual de Campinas, 1994.23p.

SILVA, G. C.; NASCIMENTO, M. T.

Fitossociologia de um remanescente de mata sobre tabuleiros no norte fluminense do estado do Rio de Janeiro (Mata do Carvão). Revista Brasileira de Botânica, v.24, p.51-62, 2001.

TABANEZ, A. A. J.; VIANA, V. M.; DIAS, A. S. Conseqüências da fragmentação e do efeito de borda sobre a estrutura, diversidade e sustentabilidade de um fragmento de floresta de planalto de Piracicaba, SP. Revista Brasileira de Biologia, v.57, p.47-60, 1997.

R. Árvore, Viçosa-MG, v.31, n.4, p.717-730, 2007 
TABARELLI, M.; MANTOVANI, W. Clareiras naturais e a riqueza de espécies pioneiras em uma Floresta Atlântica Montana. Revista

Brasileira de Botânica, v.20, p.57-66, 1999.

TILMAN, D. et al. Habitat destruction and the extinction debt. Nature, v.371, p.65-66, 1994.

TURNER, I. M.; CORLETT, R. T. The conservation value of small isolated fragments of lowland tropical rain forest. Trends in Ecology and Evolution, v.11, p.330-333, 1996.
VELOSO, H. P.; RANGEL FILHO, A. L. R.; LIMA, J. C. A. Classificação da vegetação brasileira adaptada a um sistema universal. Rio de Janeiro: IBGE, 1991. 124p.

VIANA, V. M.; TABANEZ, A. A. J. Biology and conservation of forest fragments in the Brasilian Atlantic moist forest. In SCHELLAS, J.; GREENBERG, R. Forest patches in tropical landscapes. Washington: Island Press, 1996. p.151-167. 